This is the peer reviewed version of the following article: Klippstein, Helge, Hassanin, Hany, Sanchez, Alejandro Diaz de Cerio, Zweiri, Yahya and Senevirante, Lakmal (2018) Additive manufacturing of porous structures for unmanned aerial vehicles applications. Advanced Engineering Materials, 20(9), p. 180029, which has been published in final form at https://doi.org/10.1002/adem.201800290. This article may be used for noncommercial purposes in accordance with Wiley Terms and Conditions for Use of Self-Archived Versions. 


\title{
Additive Manufacturing of Porous Structures for Unmanned Aerial Vehicles applications
}

\author{
Helge Klippstein ${ }^{1}$, Hany Hassanin ${ }^{1 *}$, , Alejandro Diaz De Cerio Sanchez ${ }^{1}$, Yahya Zweiri ${ }^{1,2}$ \\ and Lakmal Seneviratne ${ }^{3}$ \\ 1 School of Mechanical and Aerospace Engineering, Kingston University, London SW15 3DW, UK \\ 2 Visiting Associate Professor, Robotics Institute, Khalifa University of Science and Technology, P.O. Box \\ 127788, Abu Dhabi, United Arab Emirates. \\ 3 Robotics Institute, Khalifa University of Science and Technology, P.O. Box 127788, United Arab Emirate \\ [*] Corresponding author: Dr Hany Hassanin,Email:h.hassanin@kingston.ac.uk
}

\begin{abstract}
Unmanned aerial vehicles (UAVs) have shown promising benefits in many applications. This has been enabled by the emergence of additive manufacturing (AM), which give the designers a large amount of geometrical freedom. In this paper, a novel design process of fused deposition modelling (FDM) combining both topology and infill optimization is introduced for AM of high performance porous structures. Tensile testing of FDM printed samples was first carried out to study the effect of the build orientation on the mechanical properties of acrylonitrile butadiene styrene (ABS) samples. It was found that samples built perpendicular to the load axis were the weakest with a tensile strength of $29 \mathrm{MPa}$ and Young's modulus of $1960 \mathrm{MPa}$. The materials properties were fed to the finite elements analysis (FEA) for geometrical topology optimisation, aiming to maximise stiffness and reduce weight of those parts. Afterwards, an infill optimisation was carried out on the topology optimised parts using different mesostructures such as honeycomb, triangular and rectangular to achieve high structural performance. The results showed that triangular pattern with $50 \%$ infill density had the lowest developed stresses, less mass and strain energy when compared to other structures. Optimum UAVs parts of a quadcopter were successfully manufactured, assembled, and tested.
\end{abstract}

Keywords: Additive manufacturing (AM), Fused deposition modelling (FDM), Topology optimisation (TO), Acrylonitrile-Butadiene-Styrene (ABS), Infill analysis, Unmanned aerial vehicles $(U A V)$. 


\section{Introduction}

Recently, there has been a continues and growing demand for unmanned aerial vehicles (UAVs) as they find applications in many areas varying from military use to online shopping businesses ${ }^{1-4}$. Search, inspection and surveillance applications are particularly important because of the UAVs ability to work in the unknown, dirty and dangerous environments which make them more efficient when compared to conventional aircrafts ${ }^{5,6}$. Thus, the high level of confidence and the low risk of their missions trigger the continued growth of UAVs systems. In addition, technological, social and economic factors have motivated and generated funds for the development of compact and high performance UAVs. Advanced technologies offer more advantageous opportunities and considerable leverage to UAVs. High performance microprocessors, sensors, and motors systems are continually get lighter and smaller which lead to constructing UAVs with high levels of endurance and efficiency ${ }^{3,7}$. On the other hand, the advancement in the manufacturability of UAVs is another aspect that controls the development of lightweight structures. Conventional manufacturing techniques such as electrical discharge machining (EDM) ${ }^{8}$, computer numerical control (CNC) ${ }^{9}$, and lay-up composite processes ${ }^{10,11}$ were successfully used to manufacture UAVs platforms. However, they are time consuming, complex and labour intensive methods. In addition, conventional manufacturing are process oriented which restrict their ability to handle complex and lightweight UAV designs ${ }^{12}$.

Additive manufacturing (AM), also known as 3D printing, has become popular in media and attracted the public and researchers of different areas. It is continuously being understood, redefined, and bespoken to a wide range of applications such as aerospace, automotive, biomedical, and food industries ${ }^{13-17}$. Fused deposition modelling (FDM), one of the 3D printing techniques, holds great potentials in wide range of applications because of its 
availability, low cost, and good workability. In this technique, a thermoplastic filament is fed into a heating unit, extruded through a nozzle and built the desired part layer by layer according to a CAD file. FDM machines, 3D printers, are easy to access and available in different versions with affordable cost. Unfortunately, the poor mechanical properties of FDM parts restrict its full potential in manufacturing of functional parts ${ }^{18-21}$. The typical use of FDM for UAVs applications was to print UAVs prototypes. The process was found to improve the aerodynamics performance of UAVs models. Furthermore, the FDM proved to be accurate, fast, and cost effective compared to other fabrication processes ${ }^{22-25}$. Few literatures reported the use of FDM to build UAVs. Aurora Flight and Stratasys developed UAV jet using FDM of Acrylonitrile Styrene Acrylate ASA and Polyetherimide (PEI) ${ }^{26}$. They also developed vertical take-off UAV using FDM of carbon fibre ${ }^{27,28}$. Stern and Cohen ${ }^{29}$ developed a variable speed telescoping UAV for atmospheric monitoring using FDM. The UAV was fabricated using lightweight acrylonitrile butadiene styrene (ABS) structure to allow high performance aerodynamics.

The design freedoms of 3D printing are much higher than in conventional manufacturing processes. Therefore, topology optimisation becomes a very interesting design tool to obtain the best material distribution within a specific design space so that a cost function of a part is minimised. This tool was successfully implemented in many applications such as automotive, aerospace, construction, heat transfer, fluid, acoustics and others ${ }^{30}$. Typically, topology optimization is complicated as the resultant optimised geometry is often complex. In order to allow manufacturing friendly designs, a simplification of the model is required due to the production constraints. In addition, the manufacturing constraints can be also included in the topology optimization. With the merge of $3 \mathrm{D}$ printing, the manufacture of topology optimisation designs is widely enabled regardless to design complexity which does not affect 
the manufacturing cost. Moreover and in many cases, the manufacturing cost is decreased with the increase of the part complexity due to reduced material content and support structure. While the fabrication constraints of $3 \mathrm{D}$ printing processes are less important than conventional manufacturing techniques, manufacturing restriction and considerations are still need to be addressed.

While few recent literatures have introduced the use of 3D printing in manufacturing UAVs parts such as wings, tilting mechanism, and embedded electronics ${ }^{12}$, none of the reported research discussed the design methodology nor apply topology and infill optimisation of the 3D printing process for their models. This paper introduces the use of topology and infill optimisation in conjunction with FDM technology to create functional ABS parts of a quadcopter UAV. To enable the proposed topology and infill optimisation for FDM, the material properties of ABS were first obtained. Next, the topology optimization study is provided taking into consideration the FDM limitations in terms of minimum wall thickness, filaments direction and most importantly the infill strategies.

\section{Materials and Methods}

The proposed design strategy using topology and infill optimisation is shown in Figure 1. It starts with materials characterisation of the FDM samples. Next, the design space, constraints and boundary conditions of the part under investigation are defined. Topology optimisation is carried out by removing unnecessary mass and maximise the stiffness of the part. In the redesign process, the part is smoothed and further manufacturing constraints can be applied. Furthermore, infill strategy is carried out to reduce the maximum stress to weight ratio. 


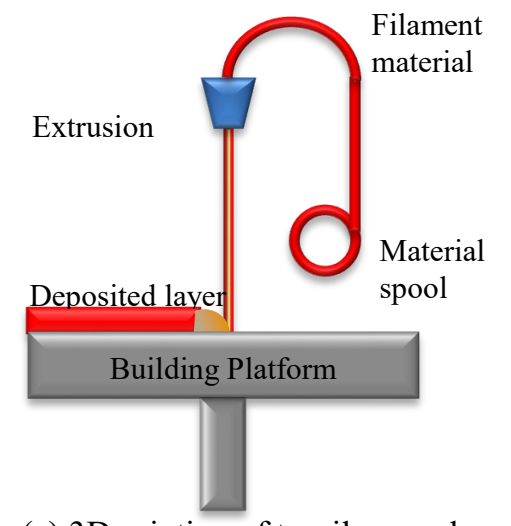

(a) 3D printing of tensile samples

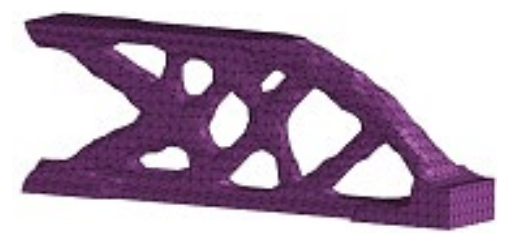

(d) Initial Topology Results

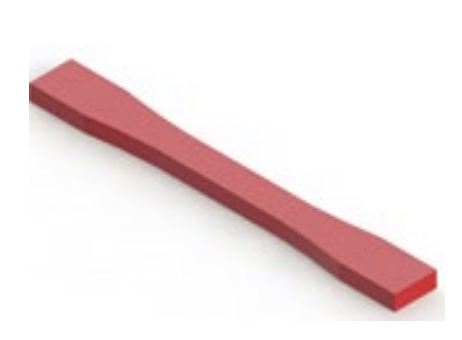

(b) Materials properties

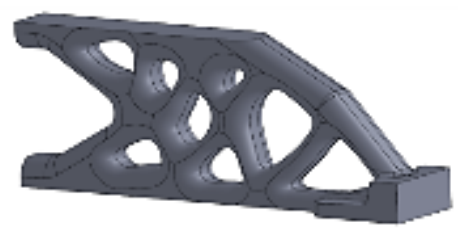

(d) Smooth the surfaces and apply manufacturing constraints

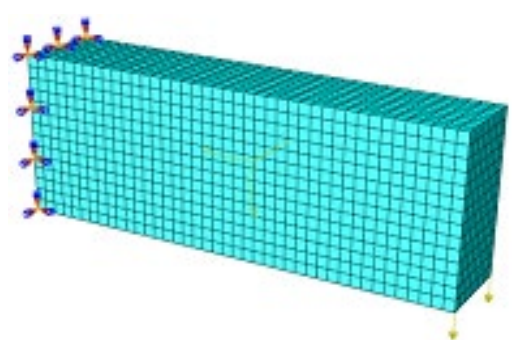

(c) Design space, constraints and boundary conditions

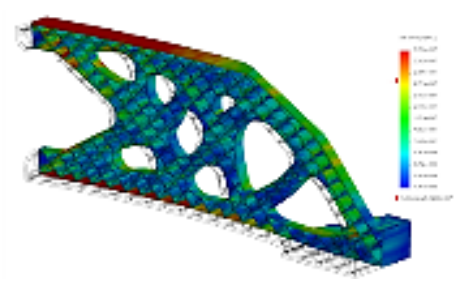

(e) FEA Infill shape optimisation

Figure 1: Design process strategy

\subsection{Tensile Test Samples and Measurements Procedure}

FDM parts show anisotropic properties based on the line by line and layer by layer manufacturing process. Therefore, it is important to investigate the material properties and feed them to the modelling process. To achieve this, tensile test measurements were carried out. Tensile test is a standard measurement technique to obtain mechanical properties such as yield strength, ultimate strength, Young's modulus, and ductility. The ABS samples started with creation of a CAD file representing the physical part, then conversion of the file using a slicing software, uploading the file to the printer, and finally printing the object. Simplify3D is the slicing software, which was used to prepare the G-code. ABS filament Z-ABS $500 \mathrm{~g}$ spool was 
supplied by (Zortrax, Poland). All the 3D printed parts were fabricated using Zortrax M200 printer with a nozzle size of $0.4 \mathrm{~mm}$, a layer thickness of 0.1 . The nozzle temperature was set as $275^{\circ} \mathrm{C}$ while the bed was set as $95^{\circ} \mathrm{C}$. Previous literatures showed that printing process parameters such as layer thickness, nozzle and bed temperature affects the mechanical properties of 3D printed samples ${ }^{31-33}$. In this study, the only difference between the samples was the sample orientating during printing. It is aimed here to investigate the material properties of two extreme building orientations. Samples fabricated with printing direction parallel to the tensile loading were designated as 0-degree printed samples. On the other hand, samples fabricated with printing direction perpendicular to the tensile loading were designated as 90 -degree printed samples. While samples fabricated with printing direction inclined with 45 degree to the tensile loading were designated as 45 -degree printed samples. The three orientations have been flat printed on the build platform. The samples have been fabricated and tested according to the ASTM D 638 standard. An extensometer epsilon model 3542 was used in the measurements to calculate the deformation of the samples during the elastic regime. The ultimate tensile strength and elastic modulus were measured and recorded for all samples. The G-code of the printing tool paths and the printed tensile test samples are shown in Figure 2. 3D printed samples during testing are shown Figure 3. The tensile testing was carried out using Zwick / Roell Z050 machine. Tensile test samples were printed and tested according to ASTM D 638 standard. An extensometer epsilon model 3542 was used, it was connected to the tensile machine to calculate the deformation of the samples. The ultimate tensile strength, ductility and elastic modulus were measured for all samples. 


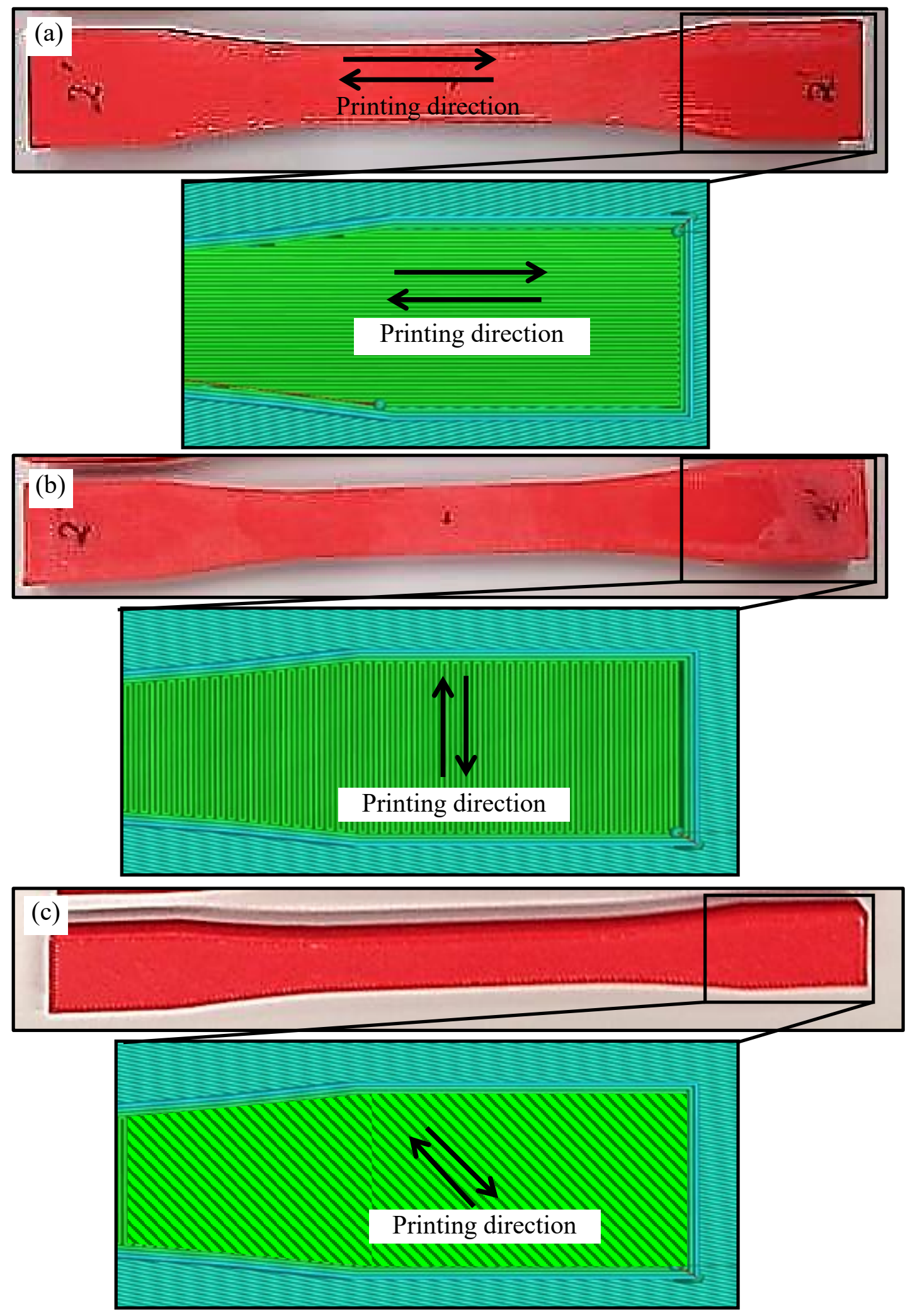

Figure 2: (a) 0-degree printed samples and G-Code of samples with longitudinal tool path, (b) 0 -degree printed samples and G-Code of samples with transverse tool path, (c) 45-degree printed samples and G-Code of samples with inclined tool path. (Green: infill, Dark blue: raft, cyan: additional raft layers) 


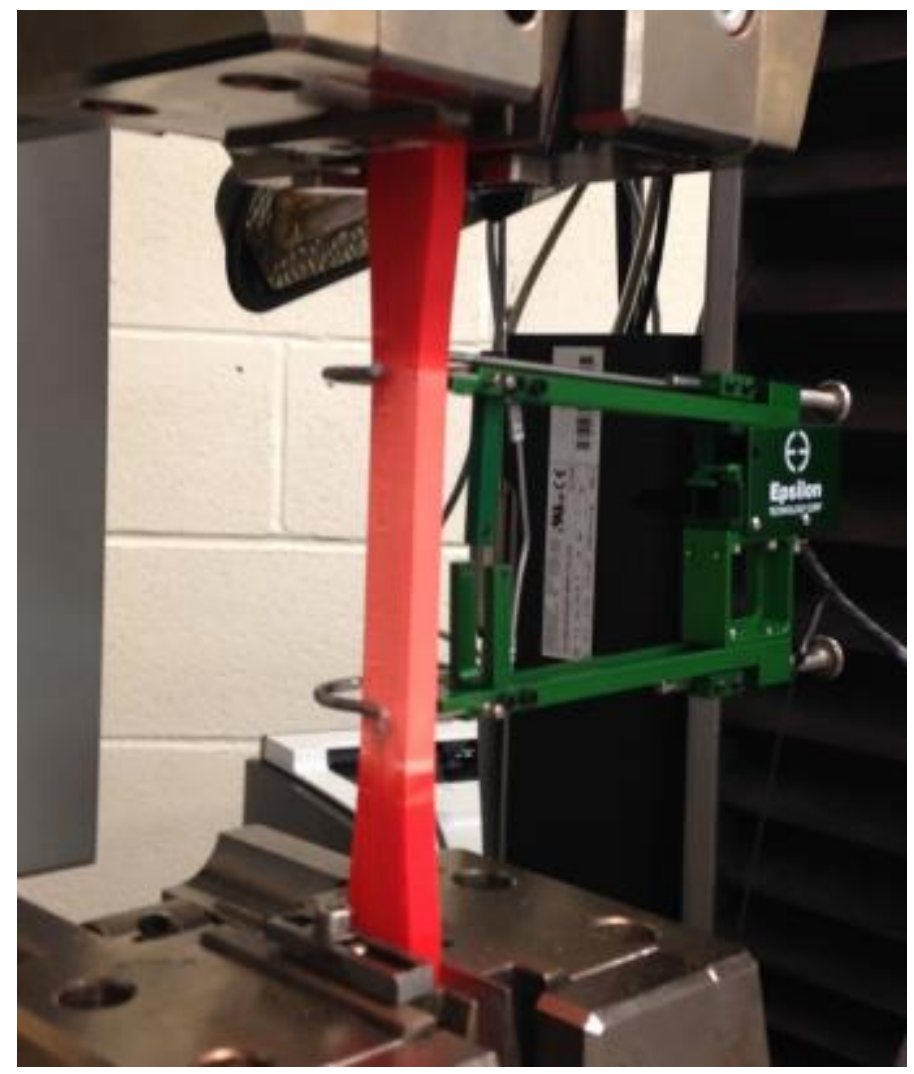

Figure 3 (a) 3D printed sample testing.

\subsection{Topology Optimisation}

The aim of the topology optimisation is to maximize the stiffness of the part. Hence, the strain energy needs to be minimized. In addition, it is also aimed to reduce the volume of the part. The total strain energy, $\mathrm{U}$ is calculated as the following with $\mathrm{V}$ as Volume, $\sigma$ as stress, $\epsilon$ as strain, $\tau$ as shear stress and $\gamma$ as shear strain.

$U=\frac{1}{2} \int_{V}\left\{\sigma_{x x} \epsilon_{x x}+\sigma_{y y} \epsilon_{y y}+\sigma_{z z} \epsilon_{z z}+\tau_{x y} \gamma_{x y}+\tau_{y z} \gamma_{y z} \tau_{x z} \gamma_{x z}\right\} d V$

Density-based topology optimization was implemented to carry out the finite element analysis of the geometry optimization. The aim of this technique is to find the best material distribution within a specific design space aiming to produce lightweight and functional UAVs parts. This 
can be achieved by discretizing the design space and obtaining the optimum density parameters associated to the discretised elements. Here, Solid Isotropic Material with Penalization (SIMP) method was used to define the relationship between the elastic modulus and the density of each element. The SIMP works by keeping a fixed finite element discretization. Each Element is then coupled with a density function $\rho(\mathrm{i})$ ranges between 0 and 1 . A density value of zero refers to a void while a density value of 1 refers to solid. Based on the density value, each element is assigned with an interpolated Young's Modulus. In order to avoid singularity failures, the assigned Young's Modulus is always larger than zero but smaller than the material Young's Modulus $\mathrm{E}_{0}$. The relationship between the elastic modulus and the density of elements can be defined as the followings:

$E_{i}\left(\rho_{i}\right)=\rho_{i}^{p} E_{0} \quad$ with $0<\rho_{i}<1$

The Young's Modulus is a function of the stiffness matrix. The intermediate density of the defined element has the penalisation factor as exponent. Elements get eliminated, as the stiffness to volume ratio becomes unfavourable for the objective function. Eventually, the constraint function is checked iteratively against the target volume $V_{\text {constraint }}$.

$\int_{\mathrm{V}} \rho(i) d \mathrm{~V} \leq V_{\text {constraint }} \quad$ with $0<\rho(i)<1$ and $i \in \Omega$

The workflow of this algorithm is based on initialisation, FEA, density function update, filtering techniques and optimisation. If the constraint converges, the process is stopped, otherwise the loops runs again ${ }^{34}$. 


\subsection{Definitions of Design Space and Boundary Conditions}

The CAD assembly of the quadcopter UAV is shown in Figure 4-a. The UAV frame consists out of four individually rotatable Carbon Fibre Reinforced Polymer (CFRP) tubes, which are mounted on the main body. The four-bar tilting mechanism is fixed on those arms and can tilt the rotor up to 10 degree inwards and 45 degree outwards. The main body holds the rotation mechanism of the quadcopter arms and all electronic parts. Each arm can rotate up to 90 degree in both directions. The overall drone weights $3.961 \mathrm{~kg}$ and has a size of $1050 \mathrm{~mm} \mathrm{x} 1050 \mathrm{~mm} \mathrm{x}$ $250 \mathrm{~mm}$.

The arm subassembly is shown in Figure 4-b. All coloured parts are originally 3D printed parts using Z-ABS. The arm assembly in green, tilting bracket in blue and arm cover cap in pink. In this paper, the titling bracket is re-designed using the topology and infill optimisation to demonstrate the effectiveness of the proposed approach. For the tilting bracket, the servo motor is represented by a rigid coupling. The motor torque and the axial load based on the four-bar mechanism are acting on the servo motor axis. The boundary conditions are shown in Figure 4-c. Only one screw located in the centre is used to hold the tilting bracket to the arm and is presented in the model as pined condition; hence, the surface is restricted to translate in $\mathrm{x}-$, $\mathrm{y}-$ and z- direction. The arm contact is simulated as cylindrical contact condition. The given four bar mechanism can be simplified as parallelogram linkage and hence the torque generated at the rotor is equal to the moment at the servo. In general, the design space should contain enough material for the algorithm to find the best solution. Figure 4-c shows also the design space for the titling bracket. The meshed design space is shown in Figure 4-d. The volume constraint is set to a target fraction value of $10 \%$ of the initial design space. Topology optimisation results with a penalisation factor of 3 had shown good results for the upcoming infill design 
improvement as it was recommended ${ }^{35}$. A minimum structure thickness of 10 times the nozzle thickness $(0.4 \mathrm{~mm})$ was chosen to ensure a good manufacturability.

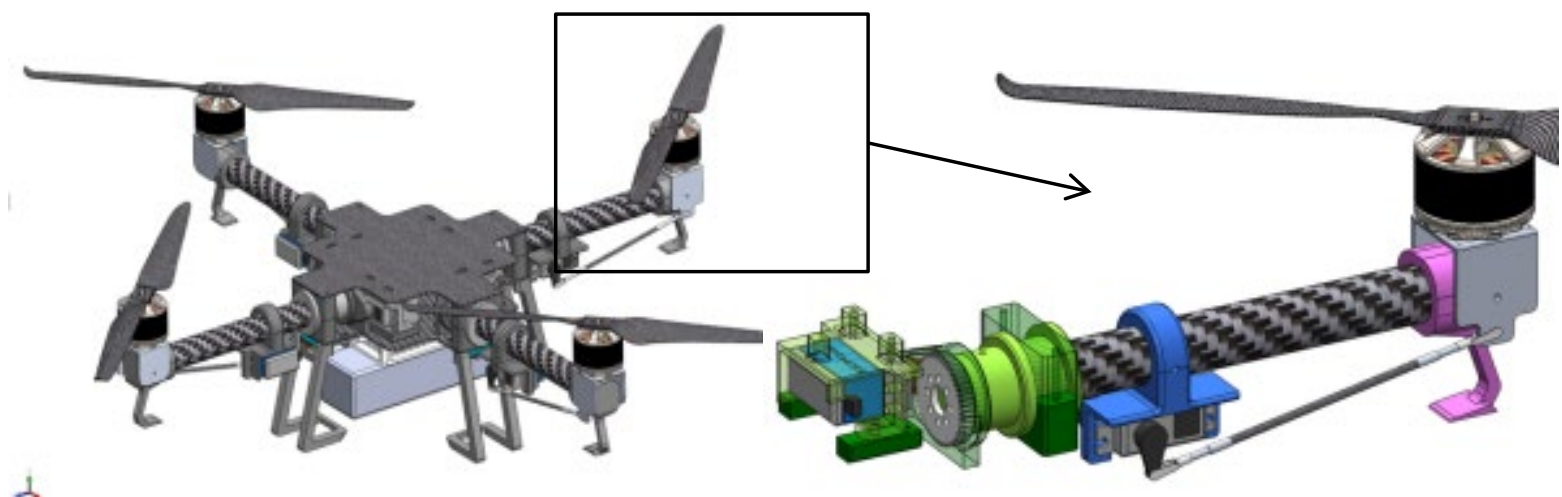

(a)

(b)

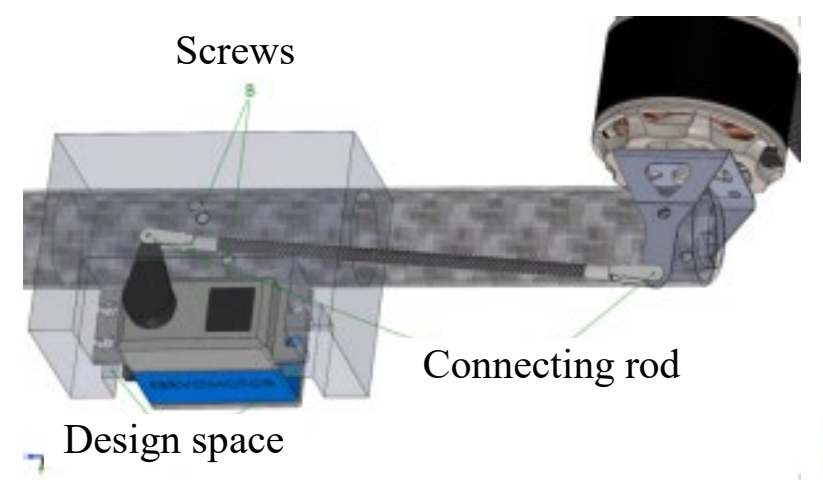

(c)

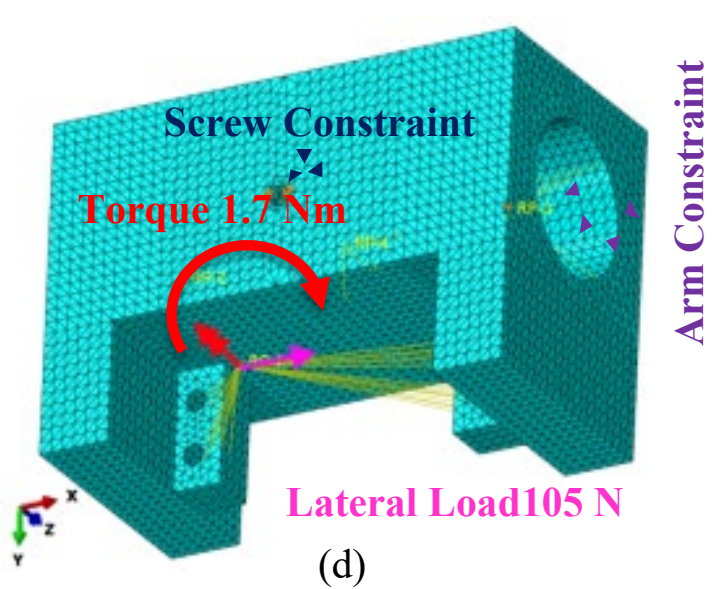

(d)

Figure 4: (a) Quadcopter Assembly, (b) Green: arm assembly, Blue: Tilting bracket, Pink: Arm cover cap, (c) Design space of the tilting bracket within arm assembly, (d) meshed design space of the tilting bracket.

\subsection{Infill Optimisation}

In FDM, the printing strategy of the interior structure of a part is referred as infill. Typically, infill is a regular shape structure, which can be chosen by the user during slicing and setting up the build using specific software. Porous structures were previously developed by a number of researchers using electron beam melting (EBM) and selective laser melting (SLM) ${ }^{36-39}$. For 
the best of the authors' knowledge, no literature was found to develop porous structures using FDM technology. The infill density and the geometry affect the physical and mechanical properties of the printed part. In general, increasing infill density leads to building stronger but heavier parts. In this study, infill optimisation was carried out to introduce porous structures into the topology optimised part in order to further reduce the weight of the UAVs parts. Honeycomb $\left(0^{\circ} /+120^{\circ} /-120^{\circ}\right)$, rectangular $\left( \pm 45^{\circ}\right)$, and triangular $\left(0^{\circ} /+60^{\circ} /-60^{\circ}\right)$ infill patterns with densities of $40 \%, 50 \%$, and $60 \%$ were used in the optimisation process, see Figure 5. Cross sections of the different infills are also shown in the figure and were investigated under optical microscope. We can observe that the honeycomb structure exhibits embedded voids as every wall consists of one layer double passes while the following layer has one pass. After introducing infill porosity into the topology optimised part, FEA calculations is carried out to investigate the consequences of using porous infill in the resultant part. 

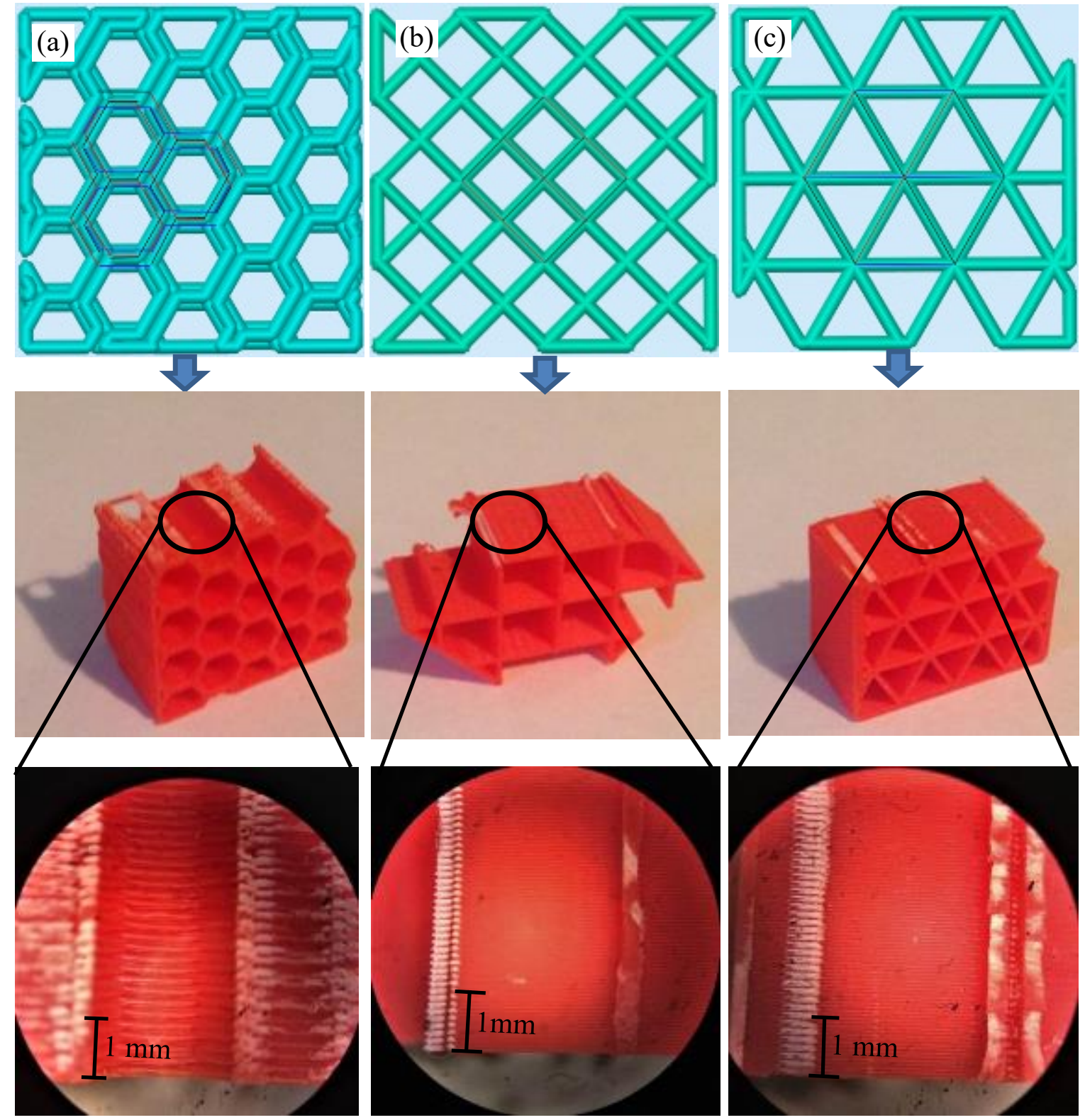

Figure 5: Slicer G-code tool path and 3D printed samples for (a) honeycomb, (b) rectangular, (c) triangular. 


\section{Results and Discussion}

\subsection{Properties of 3D printed samples}

As mentioned above the objective of carrying out the tensile test was to determine the material properties of the FDM samples. The stress strain plots of the three different samples are shown in Figure 6. The figure also shows the fracture surface of the tensile test samples. In addition, a summary of the mechanical properties obtained using tensile testing is shown in Table 1. It can be seen that 0 -degree printed samples showed ductile behaviour with a maximum strain of $18.3 \%$ while the 90 -degree printed samples showed brittle failure with a maximum strain of 2.4\%. In addition, the 0 -degree printed samples showed higher yield and ultimate strengths when compared to the 90 -degree printed samples. The properties of the 45 -degree printed samples were in the range between the 0 -degree and the 90 -degree printed samples. The better mechanical properties of 0 -degree printed samples are attributed to the orientation of the printed filaments which is aligned to the tensile test loading. This is in turn allows individual printed filaments to stretch elastically and plastically under the applied load until failure occurs. In contrast, the brittle behaviour of 90-degree printed samples owned to the delamination of adjacent filaments or layers within the samples. As presented in Figure 6 samples with 90degree infill angle confirm the brittle fracture and with no significant deformation in the gauge length. In contrast, the 90-degree infill angle samples show a vast amount of deformation indicated by the colour loss on the gauge length. The tensile testing results are consistent and in agreement with previous studies that concluded the anisotropic properties of FDM samples 40-42. The data shown in Figure 6 and Table 1 were used to the following simulation. 


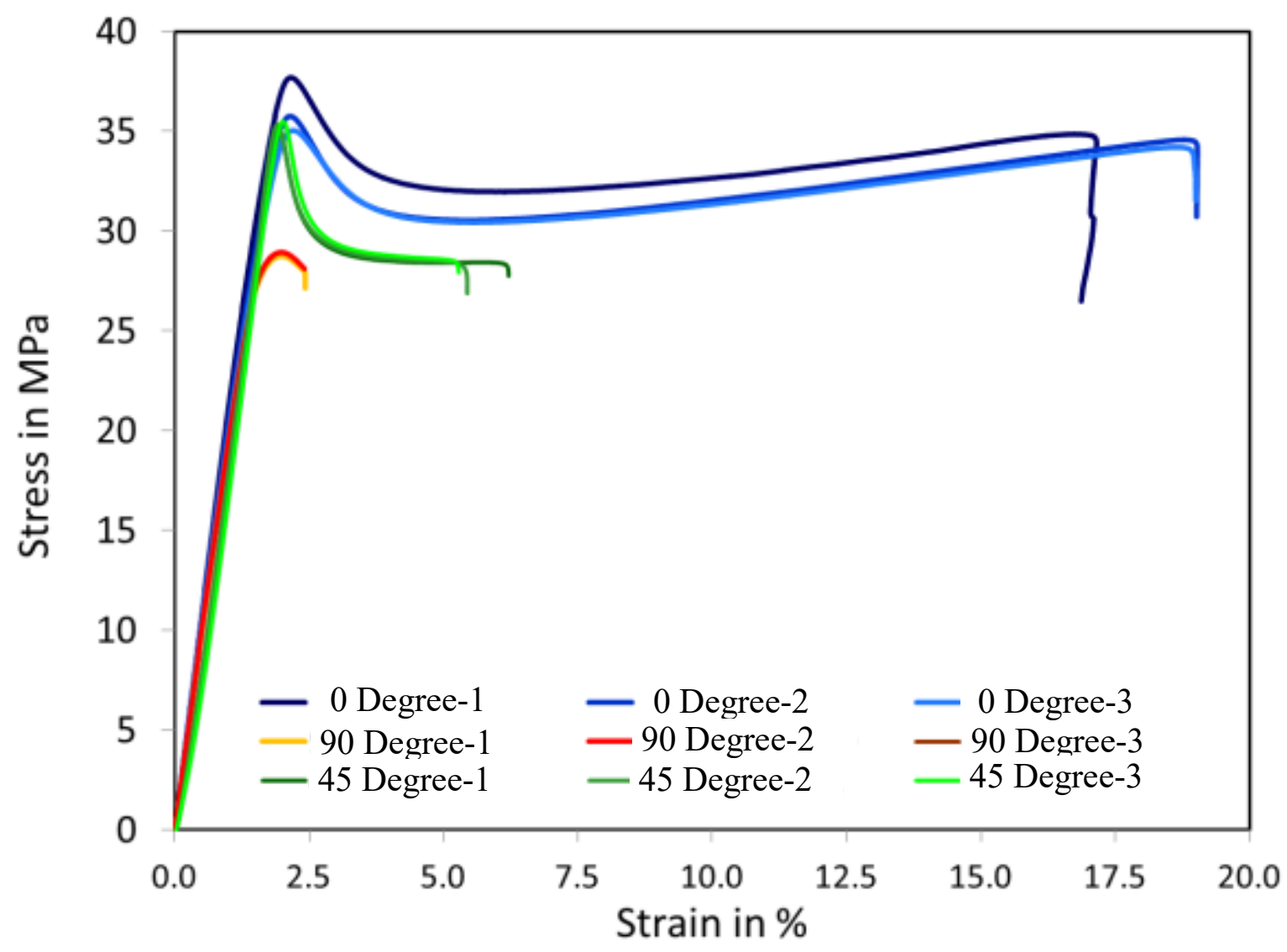

Figure 6: Stress Strain diagrams of the FDM samples.

Table 1: Mechanical Properties of the ABS FDM samples \pm standard deviation

\begin{tabular}{c|c|c|c}
\hline Infill Angle & 0 Degree & 90 Degree & 45 Degree \\
\hline Yield Strength (MPa) & $31.5 \pm 2.9$ & $27.3 \pm 0.1$ & $29.5 \pm 0.2$ \\
\hline Maximum Strength (MPa) & $35.4 \pm 1.4$ & $28.3 \pm 0.1$ & $32.2 \pm 0.1$ \\
\hline Young's Modulus (GPa) & $2.116 \pm 0.05$ & $1.963 \pm 0.02$ & $2.064 \pm 0.07$ \\
\hline Maximum Strain \% & $18.3 \pm 1.2$ & $2.4 \pm 0.0$ & $6.5 \pm 0.0$ \\
\hline
\end{tabular}




\subsection{Topology Optimisation of Tilting Bracket}

The development of the topology optimisation per step is presented in Figure 7. The figure shows the relationships between the number of cycles in the topology optimisation and both the part volume and the total strain energy. In cycle 1, the geometry shows the constraints of the fixing screws with almost no volume is assigned to the part, while in cycle 8 , with a volume fraction of $9.76 \%$ and total strain energy of $30 \mathrm{~mJ}$, the geometry shows additional ring connections around the arm at each end of the bracket. As for cycle 15 , the volume is $9.88 \%$ with total strain energy of $12 \mathrm{~mJ}$. The geometry shows wide areas, where the bracket touch the arm CFRP tube. The screw area, making the connection between the bracket and the arm has increased in size. Finally, in cycle 25 the strain energy reached a value $11 \mathrm{~mJ}$ with a volume of 9.9\% from the available design space, which shows stable and optimised value, see Figure 7. The improvement between Cycle 15 and 25 is about $1 \mathrm{~mJ}$ in total strain energy. 


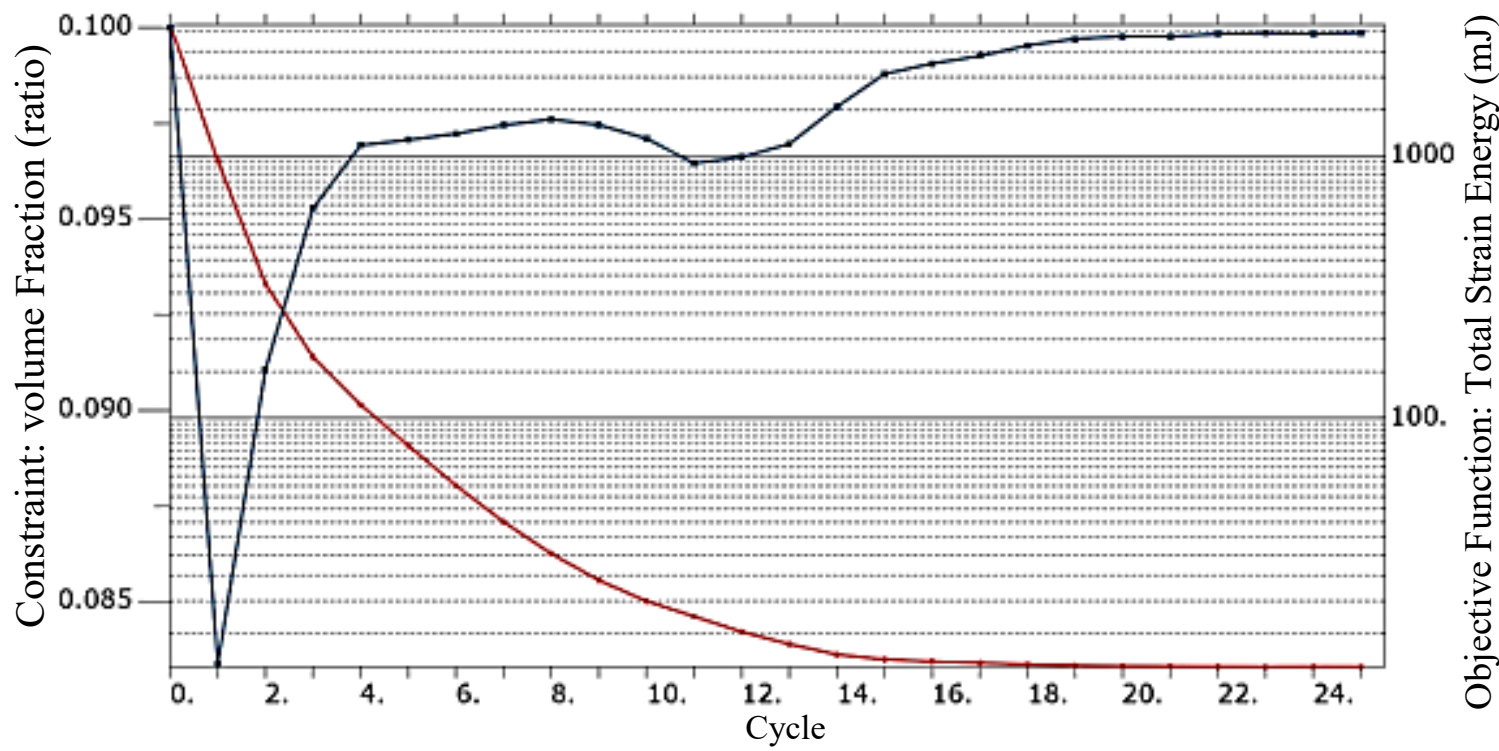

Objective Function: Total Strain Energy

Constraint Function: Volume

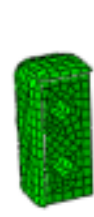

Volume $=8.33 \%$, cycle 1

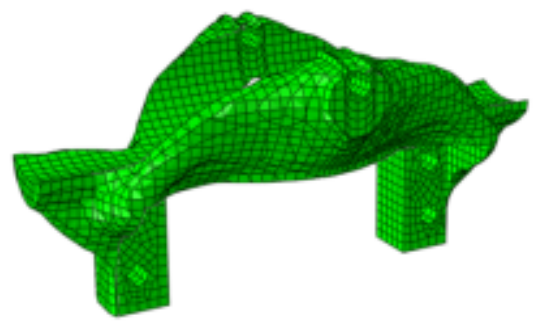

Volume $=9.88 \%$, cycle 15

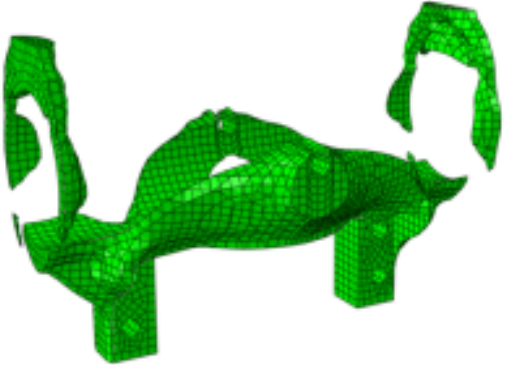

Volume $=9.76 \%$, cycle 8

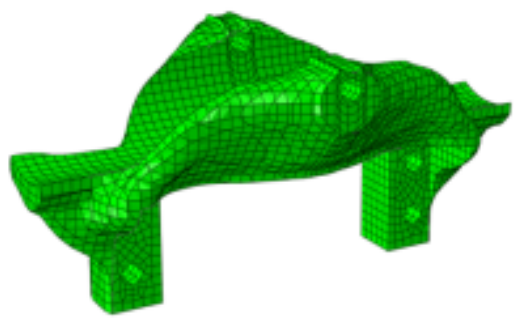

Volume $=9.99 \%$, cycle 25

Figure 7: Objective (total strain energy) and constraint (volume fraction) function. 
Topology optimised design from cyocle 25 was then further improved for the $3 \mathrm{D}$ printing by looking at the surface texture and potential weak printing of the obtained part. The FE von Mises stress analysis shows the most stresses on the front side of the bracket. The printing direction was selected to cover those stressed areas as good as possible within the tool path direction and therefore minimize the stress using 90-degree printing. The printing direction is therefore defined as 55-degree rotation around the $\mathrm{X}$ axis (Figure 8).

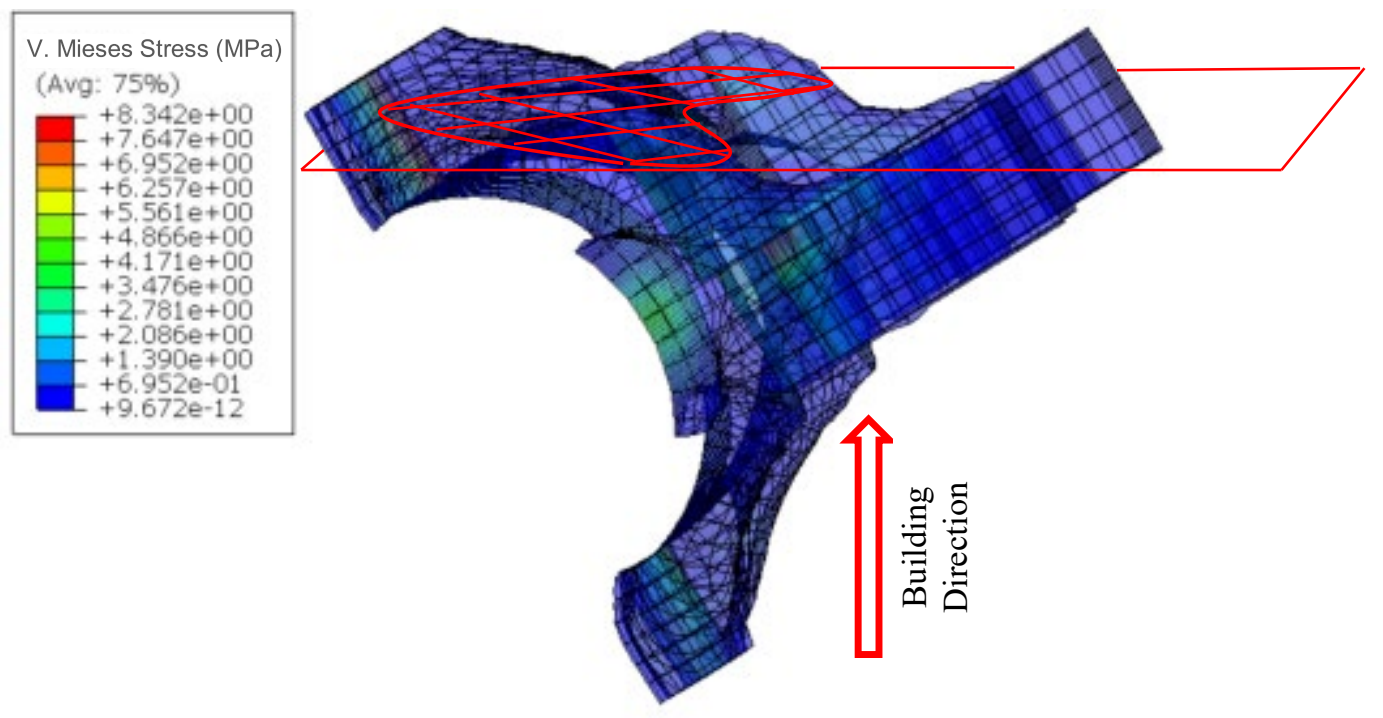

Figure 8 Tilting bracket build direction

A comparison between the original part and the Topology Optimisation (TO) geometry are shown in Figure 9. In Figure 9, the bracket is shown in Grey, while the Stereo Lithography (STL) output file of the TO is presented in Brown. The sharp edge of the functional area for the servo mount is redesigned to have smooth corners. A curved feature was implemented to generate smoother stress flow and prevent the stress concentration at the edge (Figure 9 (b) red circles). Furthermore, additional material was added on top of the top screw holes (Figure 9 (b) green circles). 
(a)

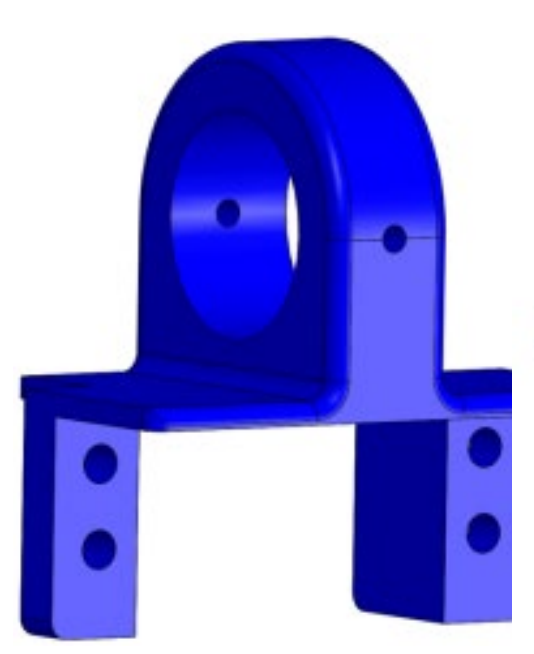

(b)

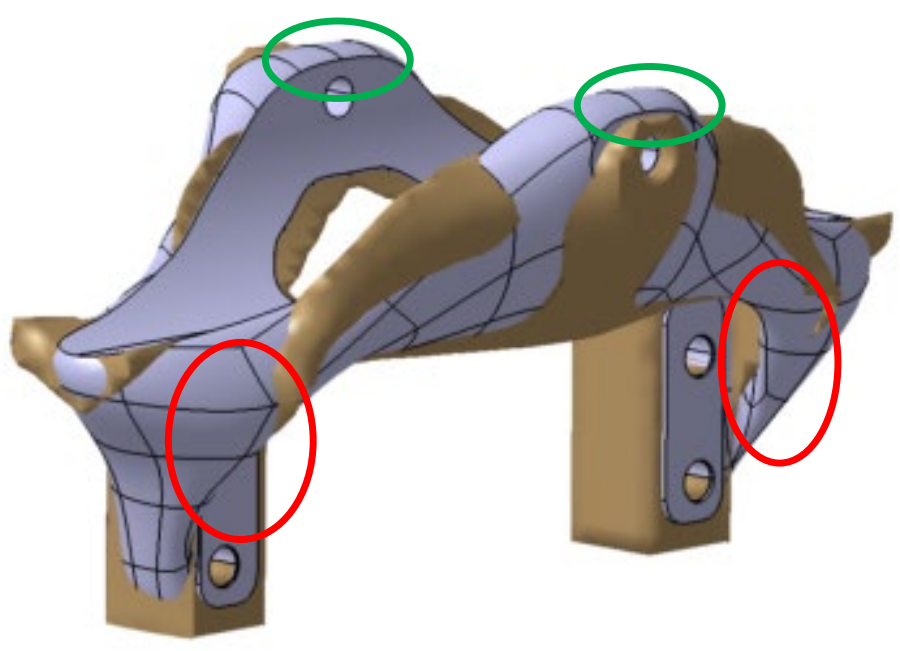

Figure 9: Tilting bracket comparison, (a) original (Blue), (b) smoothed part (Grey) with the TO results presented in Brown.

\subsection{Infill Strategies}

FEA of the modified TO part was carried out to implement different infill strategies in order to introduce porosity in the interior structure aiming to further reduce the weight of the part while maintain the strength against the applied loads. Honeycomb, rectangular, and triangular infill patterns with densities of $40 \%, 50 \%$, and $60 \%$ were used in the optimisation process. Cross sections of the slicer toolpath using honeycomb, rectangular and triangular infill patterns are shown in Figure 10. 

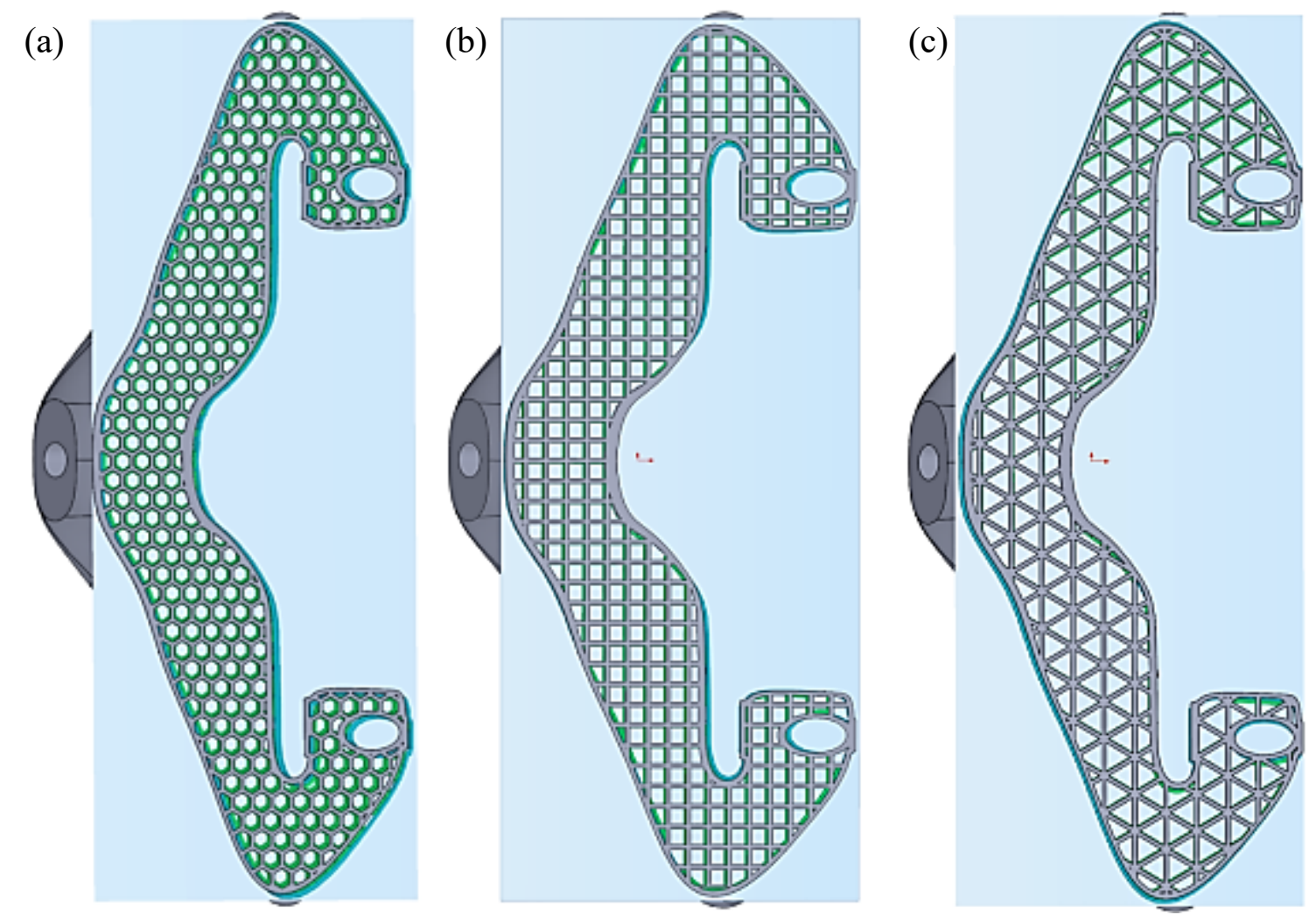

Figure 10: Comparison of slicer software tool path to CAD model (a) honeycomb, (b) rectangular, (C) triangular.

A comparison of the FEA results is shown in Figure 11. The figure shows the effect of the infill pattern and density on the FEA Von Mises stress. As expected Von Mises stress was increased with the decreasing of the infill density. In particular, the rectangular and the honeycomb infills show a significant increase in the developed maximum stresses in the $40 \%$ and $50 \%$. However, an exception was found for the triangular infill which shows lower maximum stresses at $50 \%$ than at $60 \%$ infill. A reason for this may be due to the loads and constraints are distributed differently by different infill densities. Hence, the area connecting the holes where the servomotor and the screws are mounted may vary from one infill density to another and lead to high developed stresses with a decreased infill density. 

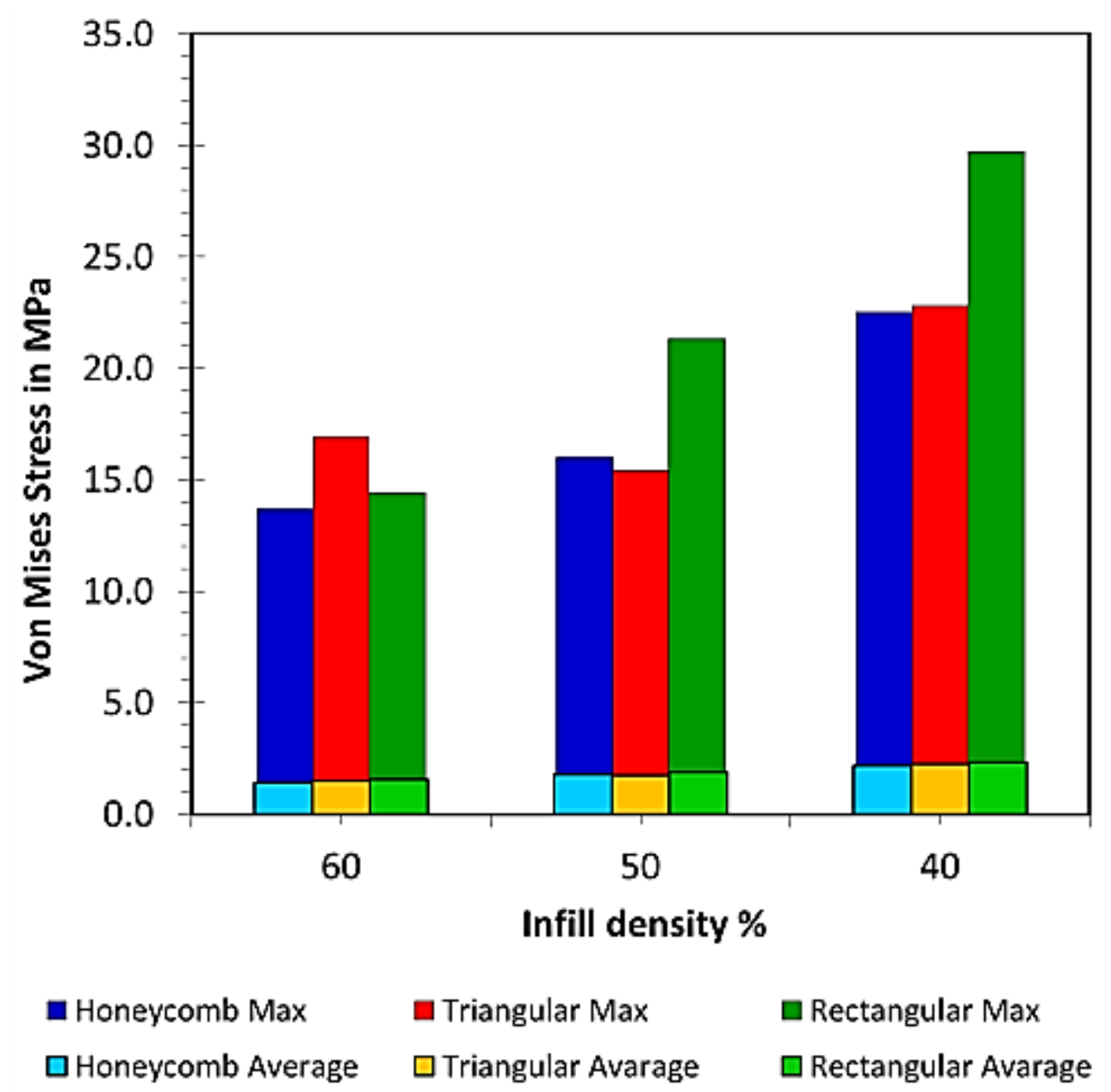

Figure 11: Effect of the infill pattern and density on the FEA Von Mises stress (maximum and average).

FEA of the $50 \%$ infill density of different infill patterns are presented in Figure 12 to Figure 13. Stress concentrations due to the infill patterns can be seen on the outer surface for each infill design. For each of the shown simulation, the highest stress concentration is in similar area around the screw holes. The section views in Figure 12 to Figure 13 (b) and (c) show the infill structure, as it supports the screw areas. This hole supporting structure is a key part for the Von Mises stress development. Based on the second moment of area, the outer surface was found to be the most stressed. Figure 11 to Figure 13 show the triangular and honeycomb with $50 \%$ infill density as they were the best compromise between the developed stresses and the 
weight reduction. However, the honeycomb structure has the potential to create defects while printing as every wall consists of one-layer double passes while the following layer has one pass, see Figure 5-a. On other hand, the triangular pattern prints the same toolpath over the layers, this reduces the chance of having any voids or errors over the printed layers, which may not have been covered by the FEA. As a result, triangular pattern with $50 \%$ infill density was used for printing the servo tilting bracket.

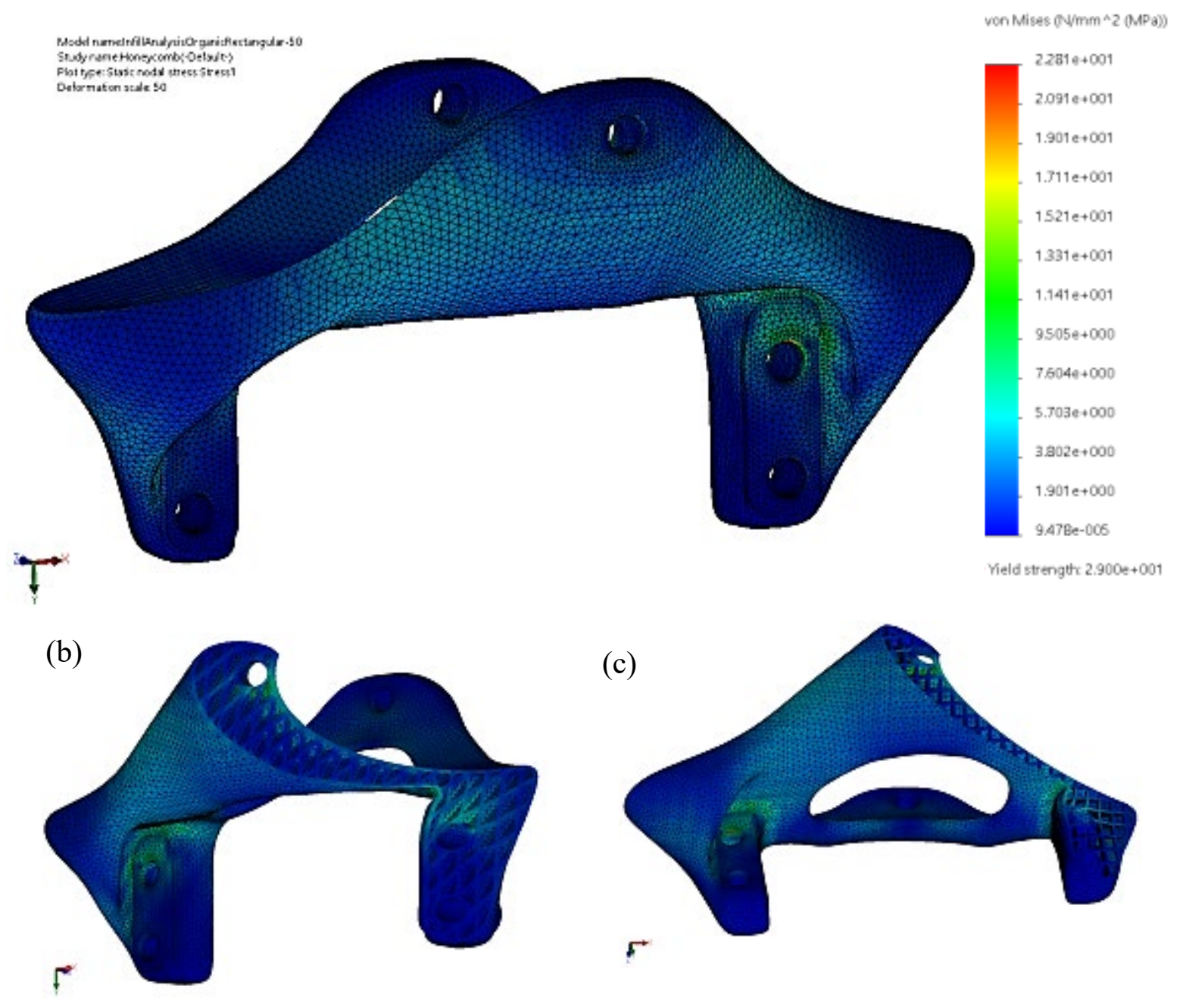

Figure 12: FEA for rectangular $50 \%$ infill pattern 
(a)

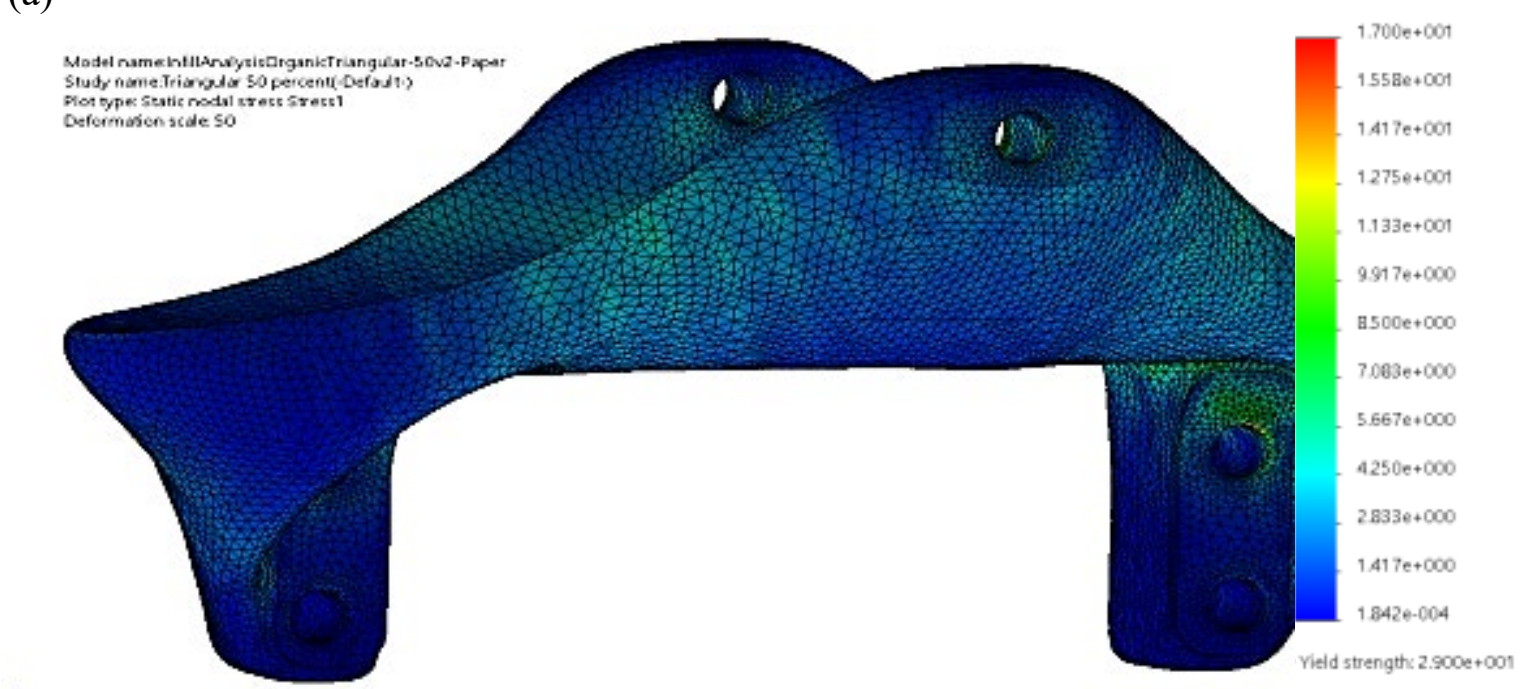

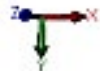

(b)

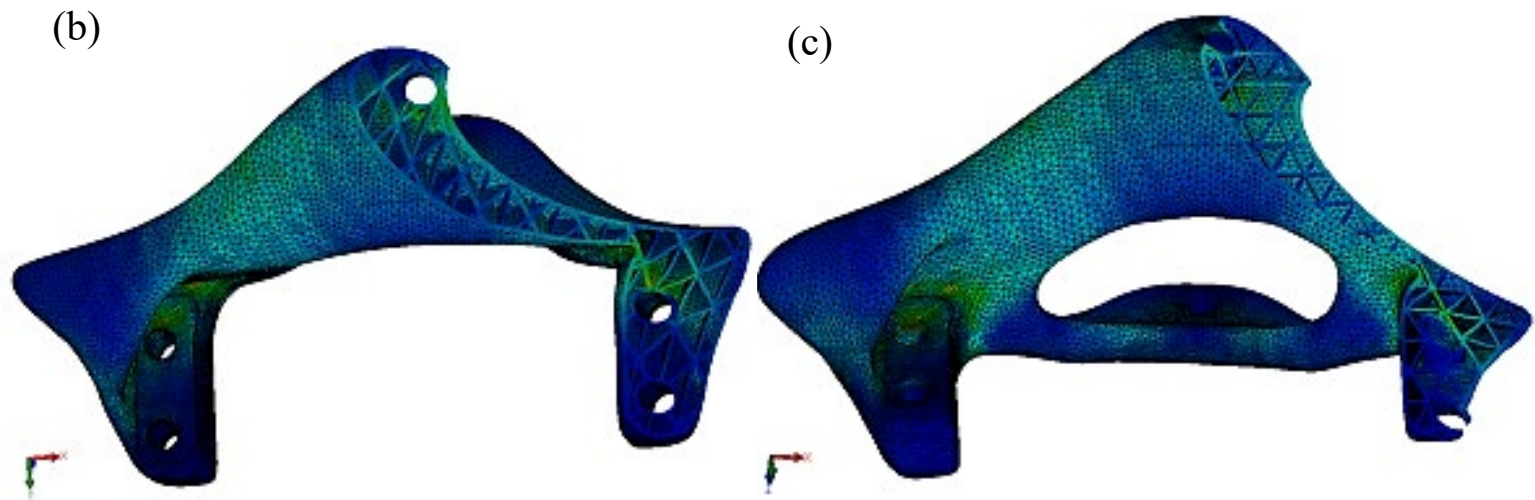

Figure 13: FEA for triangular $50 \%$ infill pattern 

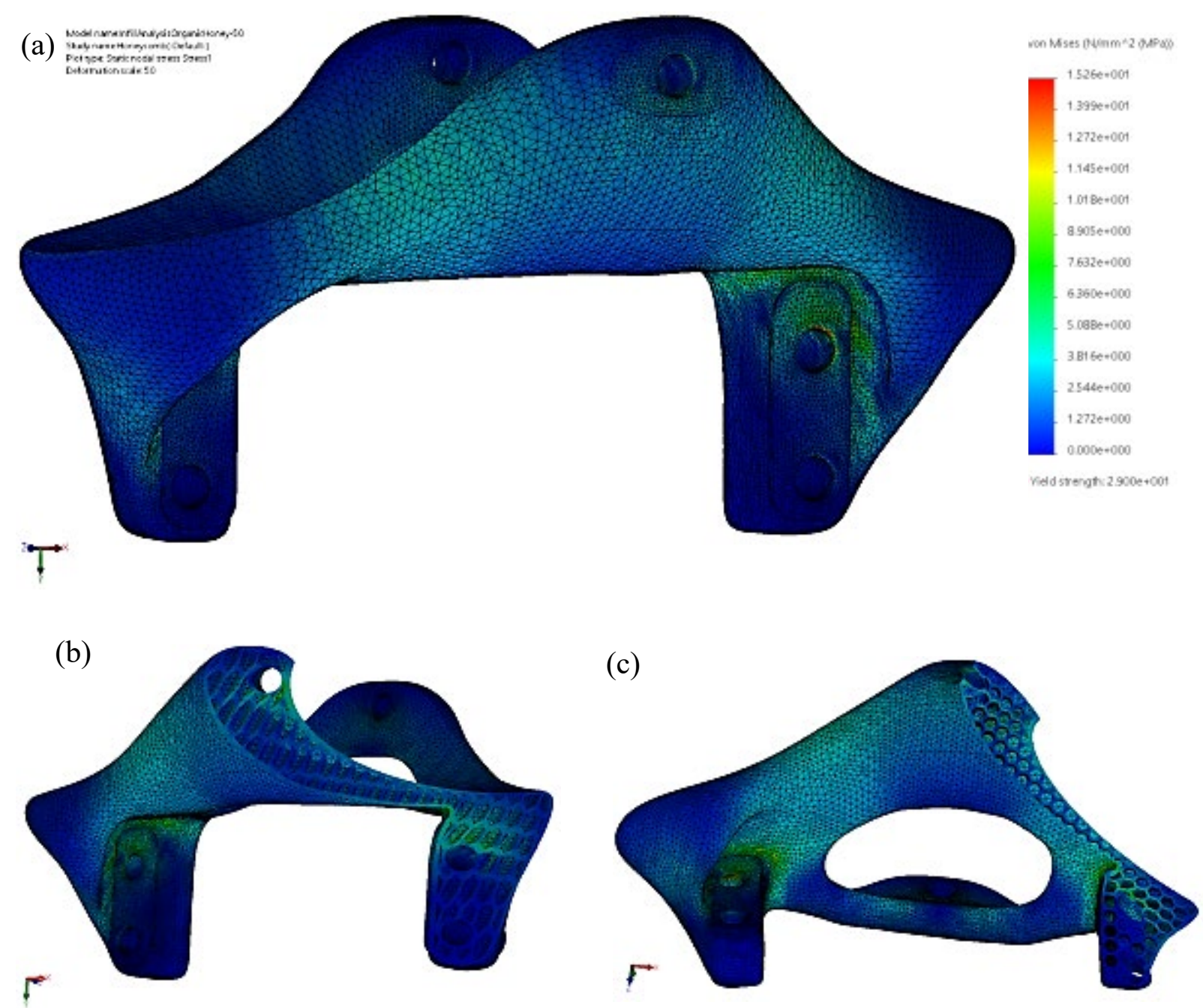

Figure 14: FEA for honeycomb $50 \%$ infill pattern.

Figure 15 shows the tilting bracket with triangular infill during printing using Zortrax FDM printer (a), as well as the final assembled state (b). A comparison between the original design and the optimised one is shown in Table 2 . The mass of the redesigned part is less than $50 \%$ of the original one while the strain energy is $11.2 \mathrm{~mJ}$ which is less than $30 \%$ of the original design. 

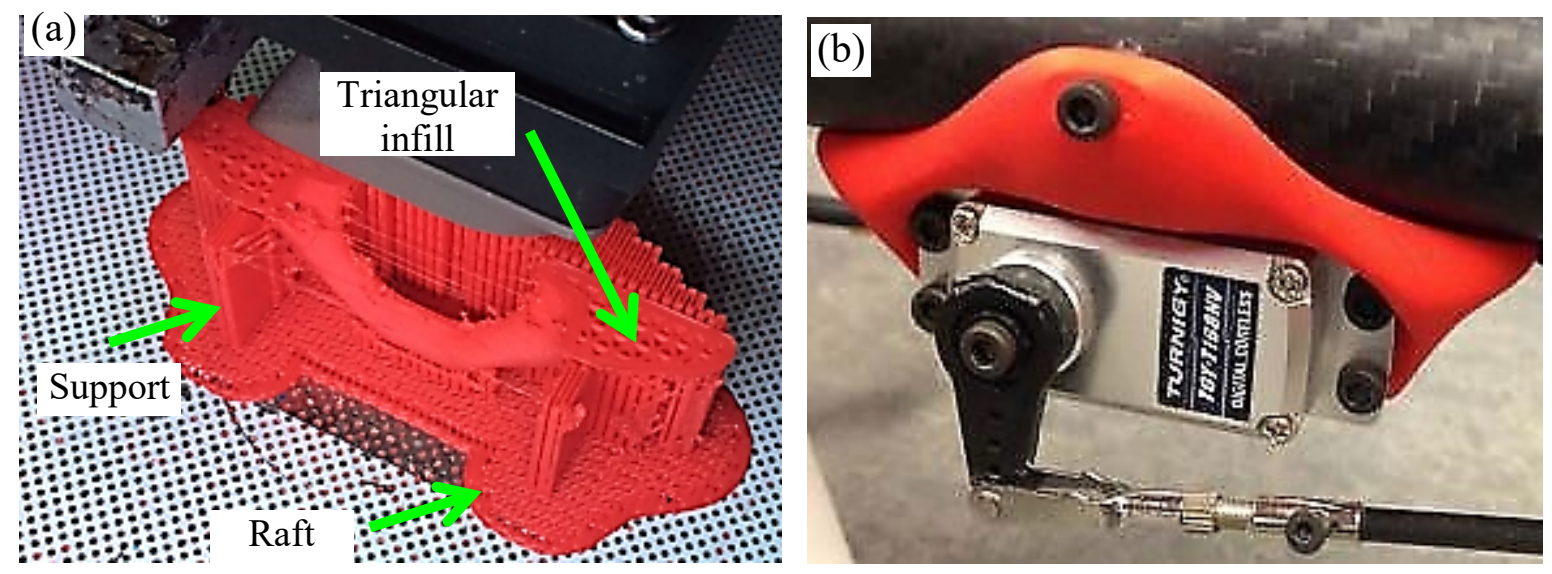

Figure 15 Tilting bracket during printing (a) and mounted in final assembly (b)

Table 2: Comparison between the original design and the optimised one.

\begin{tabular}{c|c|c}
\hline Tilting Bracket & Original & Optimised \\
\hline Mass (g) & 21.6 & 10.6 \\
\hline Percentage \% & 100 & 49.2 \\
\hline $\begin{array}{c}\text { Total Strain energy - } \\
\mathbf{1 0 0 \%} \text { dense parts (mJ) }\end{array}$ & $0.376 \mathrm{E}-02$ & $0.112 \mathrm{E}-02$ \\
\hline
\end{tabular}

\section{Assembly and Testing}

An overview of the assembly process is shown in Figure 16-a. First the arm servo is mounted between the arm rear bracket and the small counterpart. This sub assembly plus the front bracket are screwed to the upper and lower plate. The servo pin cross is put in position next. It is important to mount it in the same orientation as the arm servo connecting part in the servo 
zero position. The motor holder sheet metal part should be bolted together with the cover cap. Finally, the lateral load ring is shifted between the rear and the front bracket. The arm is introduced from the front and holds the ring in place. After fitting into the servo pin cross with the servo connector cap, the arm, ring and servo connector are bolted together, see Figure 16b. After assembling all UAV, in flight test was successfully carried out as shown in Figure 16c. The experiment has proven that the new designed parts are robust enough to withstand the loads during flight. 


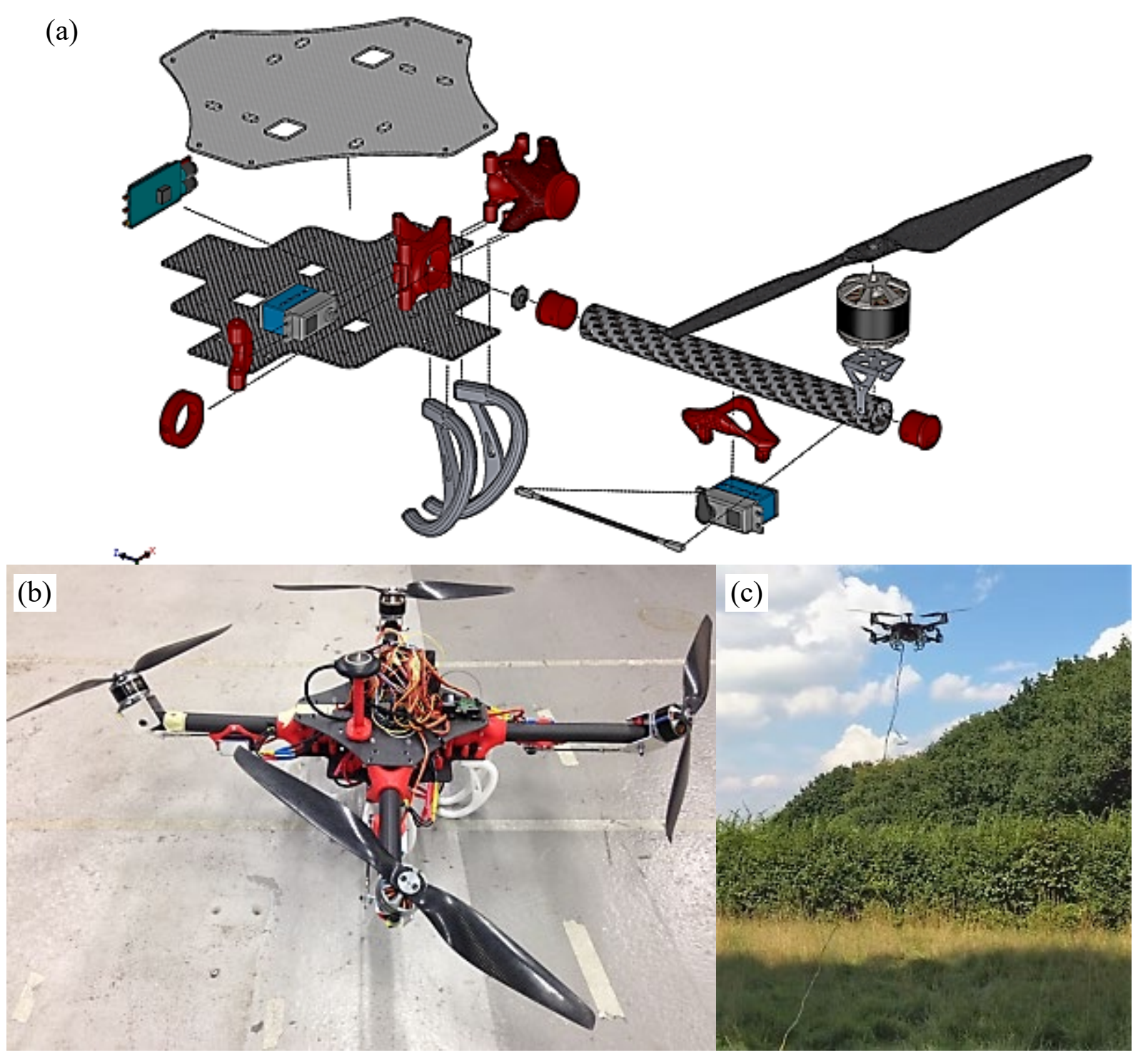

Figure 16: UAV assembly (a), fully equipped UAV including all electronics(b) and in flight test (c)

\section{Conclusion}

A 3D printing infill optimisation showed that it can be a powerful technique to bridge and link between $3 \mathrm{D}$ printing and topology optimisation in a structural and manufacturing optimization framework. The proposed methodology was applied to a servo motor tilting bracket of a quadcopter aiming to improve the design and reduce weight by introduced an optimal porous infill. Honeycomb, triangular and rectangular infill strategies were explored to complement the topology optimised part. The topology optimised part with 50\% triangular infill showed the best stress distribution, $50 \%$ mass saving and about $30 \%$ less strain energy when compared to 
the original design. As a result, functional UAVs porous structure was successfully fabricated with ease using the $3 \mathrm{D}$ printing. The experimental and in flight testing showed that the proposed methodology is efficient in re-designing functional parts for UAVs application using topology and infill optimisation which is ideal for lightweight structures such as in aerospace applications. 


\section{References}

1. Zhang, C.; Kovacs, J. M. Precision Agriculture 2012, 13,693-712.

2. $\quad$ Nex, F.; Remondino, F. Applied Geomatics 2014, 6,1-15.

3. Anderson, K.; Gaston, K. J. Frontiers in Ecology and the Environment 2013, 11,138146.

4. Yoon, J.-S.; Kim, J.; Kang, B.-S. Journal of Materials Processing Technology 2016, 233,192-205.

5. $\quad$ Fregene, K. IEEE Control Systems 2012, 32,32-34.

6. $\quad$ Sözen, V.; Craparo, E. M. Military Operations Research 2016, 21,19-35.

7. Rash, C. E.; LeDuc, P. A.; Manning, S. D., 9. Human Factors in U.S. Military Unmanned Aerial Vehicle Accidents. In Advances in Human Performance and Cognitive Engineering Research, 2006; Vol. 7, pp 117-131.

8. $\quad$ Rodrigue, H.; Cho, S.; Han, M.-W.; Bhandari, B.; Shim, J.-E.; Ahn, S.-H. Journal of Mechanical Science and Technology 2016, 30,229-236.

9. $\quad$ Cryderman, C.; Mah, S. B.; Shufletoski, A. GEOMATICA 2014, 68,309-317.

10. Alsahlani, A.; Rahulan, T.; Abdulhassan, N. International Journal of Mechanical Engineering and Robotics Research 2017, 6,71-76.

11. Liu, Z.; Zheng, X.; Feng, Y.; Zhang, D. Fuhe Cailiao Xuebao/Acta Materiae Compositae Sinica 2016, 33,1055-1063.

12. Klippstein, H.; Diaz De Cerio Sanchez, A.; Hassanin, H.; Zweiri, Y.; Seneviratne, L. Advanced Engineering Materials 20,1700552.

13. Zhang, D.; Sun, S.; Qiu, D.; Gibson, M. A.; Dargusch, M. S.; Brandt, M.; Qian, M.; Easton, M. Advanced Engineering Materials 0,1700952.

14. Li, X. Advanced Engineering Materials 0,1700874.

15. Nguyen, N.; Park, J. G.; Zhang, S.; Liang, R. Advanced Engineering Materials 0,1700876 .

16. Travitzky, N.; Bonet, A.; Dermeik, B.; Fey, T.; Filbert-Demut, I.; Schlier, L.; Schlordt, T.; Greil, P. Advanced Engineering Materials 2014, 16,729-754.

17. Felzmann, R.; Gruber, S.; Mitteramskogler, G.; Tesavibul, P.; Boccaccini, A. R.; Liska, R.; Stampfl, J. Advanced Engineering Materials 2012, 14,1052-1058.

18. Jin, Y.; Wan, Y.; Zhang, B.; Liu, Z. Journal of Materials Processing Technology 2017, 240,233-239.

19. Adel, M.; Abdelaal, O.; Gad, A.; Nasr, A. B.; Khalil, A. Journal of Materials Processing Technology 2018, 251,73-82.

20. Kaveh, M.; Badrossamay, M.; Foroozmehr, E.; Hemasian Etefagh, A. Journal of Materials Processing Technology 2015, 226,280-286.

21. Du, J.; Wei, Z.; Wang, X.; Wang, J.; Chen, Z. Journal of Materials Processing Technology 2016, 234,332-341.

22. Guerrero-Villar, F.; Torres-Jimenez, E.; Dorado-Vicente, R.; Jiménez-González, J. I. Procedia Engineering 2015, 132,78-85.

23. Rayegani, F.; Onwubolu, G. C.; Nagy, A.; Singh, H. 2014,V02AT02A010.

24. Rêgo, I. S.; Marcos, T. V. C.; Pinto, D. R.; Vilela, R. G. S.; Galvão, V. A. B.; Pivetta, A.; Camilo, G. P.; Silva, J. R. T.; Lima, B. C.; Carvalhal, A. K.; Cardoso, R. L.; Martos, J. F. A.; Santos, A. M.; Oliveira, A. C.; Toro, P. G. P. Aerospace Science and Technology 2016, 55,307-313.

25. Daneshmand, S.; Aghanajafi, C.; Shahverdi, H., Investigation of rapid manufacturing technology with ABS material for wind tunnel models fabrication. 2012; 32, p 575. 
26. Keane, P., NTU and Stratasys 3D Print Operational Drone with Embedded Electronics using Aerospace-grade Material. 2016, 2017.

27. Reisinger, D. Mini Version of DARPA X-Plane Completes Test Flight. (05/13),

28. Dahle, D. Aurora Flight Sciences Maximizes the Benefits of 3D Printed Composite Tooling. (05/13),

29. Stern, M.; Cohen, E., VAST AUAV (Variable AirSpeed Telescoping Additive Unmanned Air Vehicle). In Technical Paper - Society of Manufacturing Engineers, 2013, TP13PUB47.

30. Saadlaoui, Y.; Milan, J. L.; Rossi, J. M.; Chabrand, P. Journal of Manufacturing Systems 2017, 43,178-186.

31. Kozior, T.; Kundera, C. Procedia Engineering 2017, 192,463-468.

32. Mohamed, O. A.; Masood, S. H.; Bhowmik, J. L. Materials Today: Proceedings 2017, 4,8250-8259.

33. Mohamed, O. A.; Masood, S. H.; Bhowmik, J. L. Materials Letters 2017, 193,58-62.

34. Rouhi, M., Topology Optimization of Continuum Structures Using Element Exchange Method. 2008.

35. Tsavdaridis, K. D.; Kingman, J. J.; Toropov, V. V. Computers \& Structures 2015, $158,108-123$.

36. Zhao, S.; Li, S. J.; Wang, S. G.; Hou, W. T.; Li, Y.; Zhang, L. C.; Hao, Y. L.; Yang, R.; Misra, R. D. K.; Murr, L. E. Acta Materialia 2018, 150,1-15.

37. Zhang, L. C.; Liu, Y.; Li, S.; Hao, Y. Advanced Engineering Materials 0,1700842.

38. Qiu, C.; Yue, S.; Adkins, N. J. E.; Ward, M.; Hassanin, H.; Lee, P. D.; Withers, P. J.; Attallah, M. M. Materials Science and Engineering: A 2015, 628,188-197.

39. Zhang, L. C.; Attar, H. Advanced Engineering Materials 2016, 18,463-475.

40. Hassanin, H.; Essa, K.; Qiu, C.; Abdelhafeez, A. M.; Adkins, N. J. E.; Attallah, M. M. Rapid Prototyping Journal 2017, 23,720-726.

41. Ahn, S. H.; Montero, M.; Odell, D.; Roundy, S.; Wright, P. K. Rapid Prototyping Journal 2002, 8,248-257.

42. Sood, A. K.; Ohdar, R. K.; Mahapatra, S. S. Materials and Design 2010, 31,287-295. 